\title{
Tinnitus: pathology of synaptic plasticity at the cellular and system levels
}

\author{
Matthieu J. Guitton ${ }^{1,2 *}$ \\ ${ }^{1}$ Faculty of Medicine, Department of Oto-Rhino-Laryngology and Ophthalmology, Laval University, Quebec City, QC, Canada \\ 2 Institut Universitaire en Santé Mentale de Québec, Quebec City, QC, Canada
}

Edited by:

Jos J. Eggermont, University of

Calgary, Canada

Reviewed by:

Marco Atzori, University of Texas at

Dallas, USA

Daniel Stolzberg, University at

Buffalo, the State University of

New York, USA

${ }^{*}$ Correspondence:

Matthieu J. Guitton, Faculty of

Medicine, Department of

Oto-Rhino-Laryngology and

Ophthalmology, Pavillon

Ferdinand-Vandry local 4889, 1050,

Avenue de la Médecine, Université

Laval, Quebec City, QC G1V OA6,

Canada.

e-mail:matthieu.guitton@

fmed.ulaval.ca
Despite being more and more common, and having a high impact on the quality of life of sufferers, tinnitus does not yet have a cure. This has been mostly the result of limited knowledge of the biological mechanisms underlying this adverse pathology. However, the last decade has witnessed tremendous progress in our understanding on the pathophysiology of tinnitus. Animal models have demonstrated that tinnitus is a pathology of neural plasticity, and has two main components: a molecular, peripheral component related to the initiation phase of tinnitus; and a system-level, central component-related to the long-term maintenance of tinnitus. Using the most recent experimental data and the molecular/system dichotomy as a framework, we describe here the biological basis of tinnitus. We then discuss these mechanisms from an evolutionary perspective, highlighting similarities with memory. Finally, we consider how these discoveries can translate into therapies, and we suggest operative strategies to design new and effective combined therapeutic solutions using both pharmacological (local and systemic) and behavioral tools (e.g., using tele-medicine and virtual reality settings).

Keywords: anxiety, clinical transfer, cochlea, memory, noise-induced tinnitus, NMDA receptors, virtual reality

\section{INTRODUCTION}

Worsened in Western countries by the deleterious combination of population aging and increased noise pollution, tinnitus-the perception of sound in silence-is a major problem of public health (Nicolas-Puel et al., 2002; Nondahl et al., 2007; Belli et al., 2008; Muluk and Oguzturk, 2008). Unfortunately, and despite the fact that tinnitus strongly alters the quality of life of sufferers (Nicolas-Puel et al., 2002; Nondahl et al., 2007; Belli et al., 2008; Muluk and Oguzturk, 2008), no effective therapeutic strategy exists. Thus, tinnitus is the target of intense research by biomedical scientists investigating the physiology and the pathophysiology of the auditory system. The understanding of the neurobiological basis of tinnitus also bears major theoretical problems, which are of interest to a broad range of neuroscientists (Eggermont and Roberts, 2004; Guitton, 2006; Guitton and Dudai, 2007). Which alterations of neural systems can lead to the development of a stable phantom percept? Since tinnitus is actually an "off-line" sound, can it be compared to memory, which allows the "off-line" recall of past events? Are the neurobiological mechanisms underlying these two apparently distinct phenomena similar? In recent years, tremendous progresses in our understanding of some of the biological basis of this devastating pathology have been made (Guitton et al., 2003; Eggermont and Roberts, 2004; Guitton and Dudai, 2007).

In the last decade, animal research has clearly demonstrated that tinnitus is a pathology of synaptic plasticity (Guitton et al., 2003; Guitton and Dudai, 2007). However, the research done using animal models has also pointed to dual aspects of the biological mechanisms underlying the ontogeny of tinnitus. Indeed, if tinnitus originates into the cochlea, at the level of the synapse between the auditory hair cells and the primary auditory neurons, its perception and long-term maintenance involves complex networks in the central nervous system, both in auditory and in non-auditory (among them limbic) structures. Thus, the biology of tinnitus comprises both molecular and system-level components.

Considering the most recent experimental results, we will describe some of the biological mechanisms underlying tinnitus, specifically the phase of initiation and of long-term maintenance of tinnitus, keeping in mind the dual molecular/system aspects of this pathology of synaptic plasticity. We will then discuss these aspects from an evolutionary perspective, and present, in light of the most recent developments in tinnitus research, similarities between tinnitus and memory, to suggest theoretical frameworks to further tinnitus research. Finally, we will consider how a better understanding of the molecular and system-level mechanisms of tinnitus can translate into new, innovative, and effective targeted therapeutic strategies, both pharmacological and behavioral.

\section{MECHANISMS OF TINNITUS CELLULAR LEVEL}

The common goal of all the research performed on tinnitus is to get closer to understanding the biological mechanisms of tinnitus, in order to develop therapeutic solution to cure this pathology. Toward that end, the lingering question for most researchers in the field has been to identify the origin of tinnitus, i.e., both the anatomical location of the dysfunction that leads to tinnitus, and the molecular mechanisms underlying this abnormal 
phenomenon (Eggermont and Roberts, 2004; Eggermont, 2006; Guitton, 2006; Guitton and Dudai, 2007).

Heated debates arose in the field of tinnitus regarding the anatomical origin of tinnitus, exacerbated by the fact that tinnitus is not a single pathology, but rather a multiform symptom (see Guitton, 2006 for review). Tinnitus can appear concomitantly to hearing lost, to presbycusis, to drug intoxication, to neurinoma .... Thus, various origins could account for various forms of tinnitus. However-and despite this heterogeneity-the final result is the same (i.e., the transmission of an abnormal message through the auditory pathways), suggesting that, at least for some forms of tinnitus, a common biological basis could be identified.

Since in most cases tinnitus co-appears with problems of the inner ear, clinical evidence has suggested a peripheral origin for the majority of tinnitus (Loeb and Smith, 1967; Nicolas-Puel et al., 2002; Guitton, 2006). The cochlea-the primary auditory organ, i.e., the structure dedicated to the transduction of acoustic signals into biological neural messages-represented a candidate of interest for a "tinnitus generator." However, in order to solve this fascinating enigma, researchers were in need of appropriate animal models. Two specific sub-types of animal models of tinnitus contributed to major advances in the identification of some of the molecular pathways involved in this genesis: first, a particular form of drug-induced tinnitus-salicylate-induced tinnitus - and second, noise-induced tinnitus. When applied at high enough concentration, salicylate treatment is known to induce tinnitus in $100 \%$ of cases, characterize as being highpitch, short-term. and reversible (Cazals, 2000; Guitton et al., 2003; Puel and Guitton, 2007). Thus, salicylate-induced tinnitus represents an ideal, highly controlled, experimental model (Guitton et al., 2003; Guitton and Dudai, 2007; Puel and Guitton, 2007). However, the clinical relevance of salicylate-induced tinnitus is rather low. In contrast, noise-induced tinnitus presents a completely opposite profile. Noise overexposure produces longterm, mostly irreversible, and clinically highly relevant tinnitus. However, not all subjects (human or animal) exposed to high levels of noise will develop tinnitus (Heffner and Harrington, 2002; Guitton and Dudai, 2007).

These two opposite models of tinnitus were instrumental for understanding the biological mechanisms underlying the initiation phase of tinnitus. As an abnormal auditory perception, the biological message which is perceived as tinnitus should originate somewhere in the early neural pathways. Thus, the synapse between the sensory inner hair cells and the primary auditory neuron and the primary auditory neurons themselves are interesting candidates for the site of initiation of tinnitus. Animal models helped us to decipher how salicylate treatment could lead to the genesis of an abnormal neural signal. Indeed, salicylate is a potent inhibitor of cyclooxygenase. The blockade of this enzyme triggers an increase in the concentration of arachidonic acid in cell membranes (Guitton and Puel, 2004). Some ion channel receptors, and particularly the NMDA receptors of glutamate, are highly sensitive to the lipid composition of cell membranes. Accumulation of arachidonic acid thus mechanically "stretches" NMDA receptors, increasing their opening probability (Miller et al., 1992; Casado and Ascher, 1998). That is exactly what happens in the mammalian cochlea (Guitton et al., 2003; Guitton and Puel, 2004;
Ruel et al., 2008). Interestingly, in the cochlea, this mechanism was demonstrated first in vivo using a unique combination of pharmacological and behavioral techniques (Guitton et al., 2003, 2005; Guitton and Dudai, 2007), before being confirmed in vitro (Peng et al., 2003; Ruel et al., 2008). In vivo studies demonstrated that the local intracochlear application of NMDA antagonists was able to abolish the perception of behaviorally assessed tinnitus induced by the cyclooxygenase inhibitors salicylate and mefenamate (Guitton et al., 2003, 2005; Guitton and Dudai, 2007). In vitro electrophysiological studies confirmed that salicylate was able to specifically act on cochlear NMDA receptors both in cultures of primary auditory neurons and in cochlear slices (Peng et al., 2003; Ruel et al., 2008).

However, drug-induced tinnitus accounts for only a very limited proportion of tinnitus in humans (Nicolas-Puel et al., 2002). Clinically, the main direct cause of tinnitus is overexposure to noise (Loeb and Smith, 1967; Guitton, 2006; Nicolas-Puel et al., 2002). Noise overexposure can produce various alterations of auditory function, ranging from very subtle changes of cochlear micro-mechanics to major hearing loss and deafness (Avan et al., 2000; Kossowski et al., 2001; Le Prell et al., 2006; Ohlemiller, 2008). Overexposure to noise has also been demonstrated to trigger tinnitus in animals (Heffner and Harrington, 2002; Heffner and Koay, 2005; Guitton and Dudai, 2007). Data obtained using combined pharmacological and behavioral strategies in rats demonstrated that it was possible to extend the mechanism unveiled with salicylate-induced tinnitus to longterm noise-induced tinnitus (Guitton and Dudai, 2007). Indeed, local intracochlear application of NMDA receptor antagonists was able to prevent the occurrence of noise-induced tinnitus in $100 \%$ of cases, when applied around the time of induction of tinnitus by noise overexposure (Guitton and Dudai, 2007). This blockade was specific to NMDA receptors, as AMPA receptor antagonists, and 5-HT receptors antagonists did not prevent the onset of tinnitus (Guitton and Dudai, 2007). Furthermore, in the case of noise-induced tinnitus, the sensitivity of this process to NMDA receptor blockade remains for several days after the initial noise overexposure (Guitton and Dudai, 2007). Thus, the initial phase of both salicylate-induced and noise-induced tinnitus is dependant on NMDA receptor activity in primary auditory neurons.

\section{SYSTEM LEVEL}

The evidence that tinnitus originates from single synapses in the periphery of the auditory system does not, however, contradict the involvement of central parts of the auditory system. Sensory messages originate from the peripheral organs, but perception itself is a phenomenon subserved by system activity, i.e., subcortical and cortical neural networks. Tinnitus is not different from other sensory phenomena.

After a phase of initiation, corresponding to the ontogeny of tinnitus in the cochlea, tinnitus undergoes a phase of longterm maintenance. Likely, the biological mechanisms sustaining tinnitus during this second phase are hybrid: localized mechanisms within the cochlea, and distributed mechanisms alongside the central—auditory and non-auditory-neural networks (Eggermont and Roberts, 2004; Guitton, 2006). 
Auditory neural networks are central in the perception of tinnitus, as well as in its long-term aspects. Central auditory pathways are known to be the target of intense plasticity (Bledsoe et al., 2003). Occurrence of tinnitus triggers various forms of alteration in central auditory structures. In parallel to the research done on the molecular basis of tinnitus, intense research has been performed to unveil the neural networks affected by the presence of tinnitus (Eggermont and Roberts, 2004; Eggermont, 2006; Noreña et al., 2006).

An important phenomenon confirms the system-level involvement in the pathophysiology of tinnitus. Indeed, while the vast majority of tinnitus appears concomitantly to hearing loss, the onset of tinnitus often correlates with stressful events rather than with the onset of hearing loss (Guitton, 2006; Nicolas-Puel et al., 2002). This apparent paradox is easy to explain when considering that, under normal conditions, the human brain is able to "filter" aberrant neuronal activities propagated along auditory structures — which would be perceived as tinnitus - from the flow of pertinent sensory information. Considering the clinical reality of tinnitus, it is easy to further the analogy between tinnitus and memory, by considering that anxiety and other negative emotions act as reinforcing factors. Initially, tinnitus exists below the threshold of perception, and is thus "masked" and unperceived. Following a stressful event, a transient increase of anxiety levels triggers a lowering of the threshold of perception. Tinnitus is consequently "unmasked," and begins to be actually perceived. Finally, the perception of tinnitus triggers more anxiety, which in return reinforces tinnitus (Figure 1). Data obtained in animal models showing that anxiety induced by a serotonergic agent was able to exacerbate the perception of tinnitus support this system-based mechanism (Guitton et al., 2005).

The relationship between tinnitus and anxiety strongly suggests that, among the non-auditory pathways, the limbic system is involved in the long-term maintenance of tinnitus (Guitton et al., 2005; Guitton, 2006). For instance, salicylate treatment known

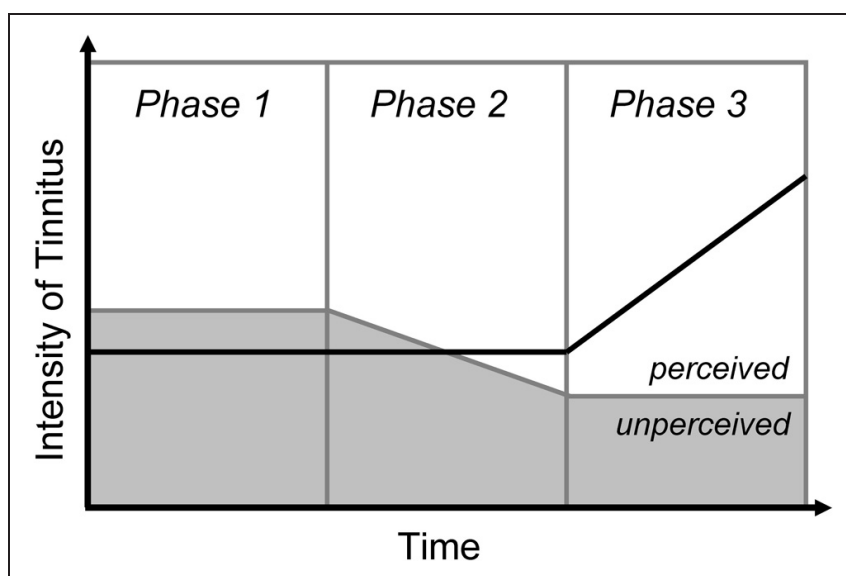

FIGURE 1 | Modulation of the perceived intensity of tinnitus by anxiety. Phase 1: Tinnitus exists below the threshold of perception, in an unperceived state. Phase 2: The occurrence of a stressful event lowers the threshold of perception. Tinnitus thus gets unmasked, and begins to be perceived. Phase 3: Tinnitus triggers anxiety, which in turn reinforce the perception of tinnitus, leading to a "vicious circle." to induce tinnitus elicits plastic changes in limbic structuresnotably in the amygdala-in addition to central auditory structures (Wallhäusser-Franke et al., 2003; Mahlke and WallhäusserFranke, 2004). Furthermore, the limbic system — the "emotional brain" - can be strongly activated by perceptual cues, leading to powerful conditioning, such as auditory fear conditioning (Apergis-Schoute et al., 2005; Ben Mamou et al., 2006). Thus, the neuronal circuitry underlying the link between auditory memories and emotional systems exists within the brain, providing a biological substrate for a limbic modulation of off-line auditory information.

One of the effects of this mobilization of central systems, whether related or not to the primary auditory pathways, is the impact of tinnitus on social behaviors. Indeed, tinnitus is often accompanied by significant alterations of high-level cognitive functions, particularly inter-individual and social interactions. Such alterations clearly add to the burden of tinnitus on the patients and their families, and strongly impair the professional activities of the sufferers. Similar effects have been demonstrated in animals, using standardized assessment of social behavior (Guitton et al., 2008; Guitton, 2009). When perceiving tinnitus, animals exhibit striking alterations of their social behavior: tinnitus-perceiving animals actively seek inter-individual contact, but once the social interactions are initiated, they are unable to sustain them (Guitton, 2009).

\section{EVOLUTIONARY PERSPECTIVES: SIMILARITIES WITH MEMORY \\ CELLULAR LEVEL}

Since evolutionary processes favor the selection of the most efficient phenotypes, how could the conditions for a synaptic pathology such as tinnitus have emerged in the highly evolved mammals' auditory system? The biological mechanisms of tinnitus, as described earlier, calls for a direct comparison with memory, both at the molecular and at the system-levels. Memory-the retention of information over time-is considered to be supported at the biological level by the plasticity of neurons and neuronal structures.

Although normal neurotransmission between the inner hair cells and the primary auditory neurons involves AMPA receptors of glutamate and not NMDA receptors, NMDA receptors still exist in the mammalian cochlea, and are activated under certain conditions, e.g., during the regrowth process after excitotoxic injury (d'Aldin et al., 1997). Thus, auditory neurons still have the potential for NMDA receptor dependant structural plasticity.

With its nature of NMDA receptor dependant mechanism, and its temporal pattern, the slow synaptic plasticity associated with the initiation of tinnitus shares striking similarities with what is observed in memory, especially during the consolidation period (Shimizu et al., 2000; Dudai, 2004; Ben Mamou et al., 2006; Takehara-Nishiuchi et al., 2006). Both phenomena are dependant on NMDA receptors containing the $2 \mathrm{~B}$ subunit (NR2B). Indeed, the involvement of this particular subunit is known to be critical for memory formation (Nicoll and Malenka, 1995; Rosenblum et al., 1996; Sotres-Bayon et al., 2007). Similarly, studies in animal model have demonstrated that a NR2B-targetted blockade of cochlear NMDA receptor activity using the pharmacological 
agent ifenprodil - a NR2B antagonist-was sufficient to abolish the perception of long-term noise-induced tinnitus in rats (Guitton and Dudai, 2007). Another cue lies in the fact that salicylate treatment known to induce tinnitus triggers modifications of the expression of the transcription factor c-fos (WallhäusserFranke et al., 2003; Mahlke and Wallhäusser-Franke, 2004), which is considered to be one of the markers of a memory-related form of neural plasticity (Reijmers et al., 2007). In addition, salicylate treatment induces changes in the expression of the transcription factor CaRF1, involved in the regulation of BDNF (Singer et al., 2008). Accordingly to molecular similarities between tinnitus and memory (Guitton and Dudai, 2007), this consolidation-like period should correspond to a phase in which tinnitus is still labile, i.e., a period during which tinnitus could be erased by pharmacological agents, as it has been demonstrated for nonconsolidated memory in other systems (Berman and Dudai, 2001). Indeed, during this period, tinnitus can be abolished by cochlear application of NMDA antagonists (Guitton and Dudai, 2007), akin to what is observed in memory (Berman and Dudai, 2001).

Primary auditory neurons seem to share with cortical neurons the same NMDA-dependant plasticity (Guitton and Dudai, 2007). Whereas in cortical neurons this property leads to the formation of memory, in the periphery, it can lead to the initiation of tinnitus (memory of a sound ...). Finally, memories are dynamic and may enter anew into a labile state when retrieved, in a process called reconsolidation (Dudai, 2006). Tinnitus could actually undergo the same fate, or be in a state comparable to "constant-reconsolidation." Given the fact that consolidated auditory fear memories have been shown to be dependant on NMDA receptors activity (Ben Mamou et al., 2006), this last comparison between tinnitus and memory opens important avenues in the development of new therapeutic strategies to cure tinnitus, as well as in our understanding of some of the biological mechanisms underlying memory.

\section{SYSTEM LEVEL}

If the first stage of tinnitus could be qualified as a consolidationlike process, what is the fate of tinnitus once this critical period is over? This question of long-term maintenance of tinnitus is critical for the understanding of this pathology, and for the development of therapeutic strategies. A first possibility would be that tinnitus "stays" in the periphery, but under the dependence of other molecular pathways. A second hypothesis, which bears fascinating conceptual avenues, is that over time tinnitus progressively recruits several anatomical structures (Figure 2) - the peripheral auditory system, the central auditory system, the limbic system, and higher-order brain structures (Eggermont and Roberts, 2004; Eggermont, 2006; Guitton, 2006; Guitton and Dudai, 2007). This distribution of the tinnitus engram from one location to multiple locations strongly echoes the process of system-level consolidation which appears in memory (Dudai, 2004, 2006). From an initial location-the cochlea for tinnitus, and the medio-temporal lobe for different forms of memory (Dudai, 2004) - the engram moves forward to complex networks of structures within the brain (Dudai, 2004). This second hypothesis would thus explain how tinnitus with a peripheral

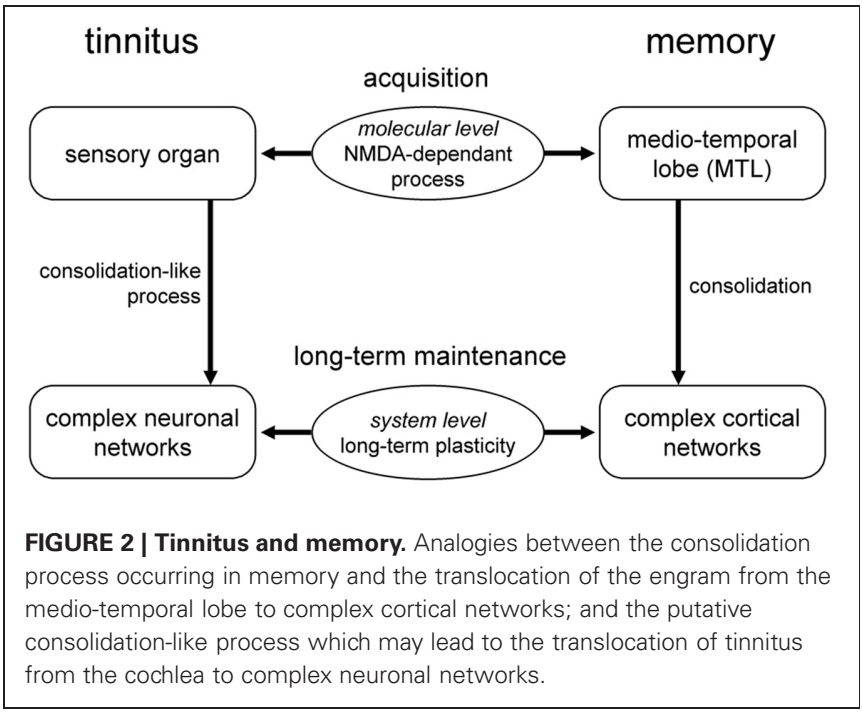

origin may undergo centralization (Figure 2). The significant plasticity observed along the auditory structures after acoustic trauma-evidenced both at the molecular (Milbrandt et al., 2000; Wallhäusser-Franke et al., 2003; Mahlke and Wallhäusser-Franke, 2004) and system-levels through electrophysiological recordings (Willott and Lu, 1982; Kimura and Eggermont, 1999; Kaltenbach et al., 2004; Eggermont, 2006; Noreña et al., 2006)—may partly account for the translocation of the engram corresponding to tinnitus.

This comparison between the neuronal substrates of tinnitus and those of memory could also be extended with another "offline" percept: chronic pain. In this case, the two phenomena also share striking similarities at the molecular level, and a parallel fate at the system-level. The early comparison between tinnitus and "phantom limb" sensation, though not completely appropriate as tinnitus is not defined by the perception of a sound in the context of total hearing loss, may still contain interesting elements for neuroscientists to explore. Recent data suggested that it is possible to reverse some of the functional brain abnormal activity responsible for the perception of tinnitus in animals by using brief electrical stimulations of the neural circuitry (Engineer et al., 2011). Of course, direct translation of these experiments in humans could be problematic, as "erasing" neural activity in auditory structures could have deleterious impact on functions such as language. Furthermore, the specificity of such approach could be questioned. However, such results strongly reinforce the notion of system involvement in the physiopathology of tinnitus.

\section{THERAPEUTIC IMPLICATIONS CELLULAR LEVEL}

Identification of defined molecular pathways, in a specific cellular type and in a constrained anatomical location, allows for the development of targeted pharmacological strategies. The development of finely targeted therapeutic strategies is one of the key trends of modern research in pharmacology. There are numerous advantages of targeted strategies, including the decrease in the drug concentration required to obtain a therapeutic result and the diminution of unwanted side effects. However, the in situ real life 
application of such strategies is often bound by multiple practical problems. Indeed, even drugs specifically designed to act on a particular receptor often have a reverse side. For instance, in highly modulated biological systems, blocking one single sub-type of a receptor may not be enough to block the biological function, as compensatory mechanisms may take place. In addition, even the most particular sub-type of a receptor may exist in different biological networks within an organism. Furthermore, despite the targeting of a particular receptor, the limited expression of this receptor may require the use of large concentrations of pharmacological agents, thus loosing part of the advantage of using targeted strategies.

Such difficulties, however, seem to be less marked in the case of tinnitus. Indeed, the cochlea offers all the characteristics of an appealing target for organ-directed pharmacological approaches. Due to its morphological characteristics, the cochlea is a natural chamber of perfusion. The sensory hair cells are surrounded by liquids which are easily reachable through the round window membrane via a trans-tympanic approach. Furthermore, the contacts between the cochlear fluids and the cephalo-spinal fluid are extremely limited, allowing the use of pharmacological agents without risking the contamination of other neuronal structures.

As mentioned above, both salicylate-induced and noiseinduced tinnitus can be blocked in animals by local (cochlear) application of NMDA antagonists (Guitton et al., 2003; Guitton and Dudai, 2007). Local application allows the use of very small doses of pharmacological agent, without major side effects in the brain. The time window of sensitivity to NMDA antagonists is several days after the trauma itself, which provides a long enough time to act to cure tinnitus. However, local organ-targeted molecular pharmacology approaches could still be used after these first several days following the insult. Indeed, as NMDA receptors seem to be common molecular integrators at the first stages of tinnitus, other molecular pathways could be involved in the later plastic changes underlying tinnitus. The molecular pathways critically involved in pathophysiological mechanisms of auditory structures represent appealing candidates for pharmacological targets to cure long-term tinnitus. Among them, cytoskeletal plasticity, with proteins such as Microtubule-associated Proteins (MAPs) or activity-dependent cytoskeletal protein (Arg3.1, also known as Arc) represents an interesting molecular pathway to investigate (Ladrech et al., 2004; Panford-Walsh et al., 2008). MAP has been reported to play a key-role in several pathophysiological conditions in the cochlea, ranging from synaptic reorganization following noise overexposure in the cochlea (Ladrech et al., 2004), to reaction to aminoglycoside toxicity (Ladrech and Lenoir, 2002). Expression of several MAP isoforms (in particular the MAP2c isorform, known for its role during the development of neurons) appears to be tightly regulated during the repair processes that occur in primary auditory neurons after excitotoxic injury in the cochlea, as well as after cochlear intoxication by amikacin, one of the well-known ototoxic drug (Ladrech and Lenoir, 2002; Ladrech et al., 2004). In this last case, the MAP pathway has been suggested to play a key role in the survival of the remaining damaged sensory cells (Ladrech and Lenoir, 2002). Results obtained on animal models of salicylate-induced tinnitus demonstrated change of Arg3.1 and
BDNF during salicylate treatment in auditory structures, reinforcing the interest of cytoskeleton proteins as potential targets of research for tinnitus (Panford-Walsh et al., 2008; Singer et al., 2008). An alternative way to look at this problem of dysfunction of cochlear plasticity in tinnitus is to modulate the GABAdependant inhibition in the cochlea. Recent works demonstrated that intra-cochlear application of midazolam [a GABA(A) receptor modulator] resulted in the reversion of salicylate-induced perception in animals (Panford-Walsh et al., 2008).

Finally, similar molecular approaches that aim at other structures of the auditory pathways could be developed. The inferior colliculus, which has the advantages of being a relatively early subcortical structure of the auditory pathways, and of being the seat of significant levels of plasticity, represents a very interesting target for pharmacologically-based therapies (Suga et al., 2000; Bledsoe et al., 2003; Guitton, 2006). Thus, molecular targeting to cure tinnitus could aim not only the periphery, but also at the central auditory structures.

\section{SYSTEM LEVEL}

As stated above, discoveries made-and to come-of the molecular pathways involved in the generation and maintenance of tinnitus will lead to important therapeutic applications. However, understanding and recognizing the system-level aspect of tinnitus also bears promise for the development of possible therapeutic strategies to cure tinnitus. It particularly allows for the optimization of the design of advanced therapeutic strategies (Figure 3). Although non-pharmacological strategies cannot abolish the perception of tinnitus, system-based strategies have been proven to have some effectiveness in helping sufferers to relieve some of the symptoms of tinnitus. In other words, some system-based strategies (masking, behavioral therapies) can help the sufferers to

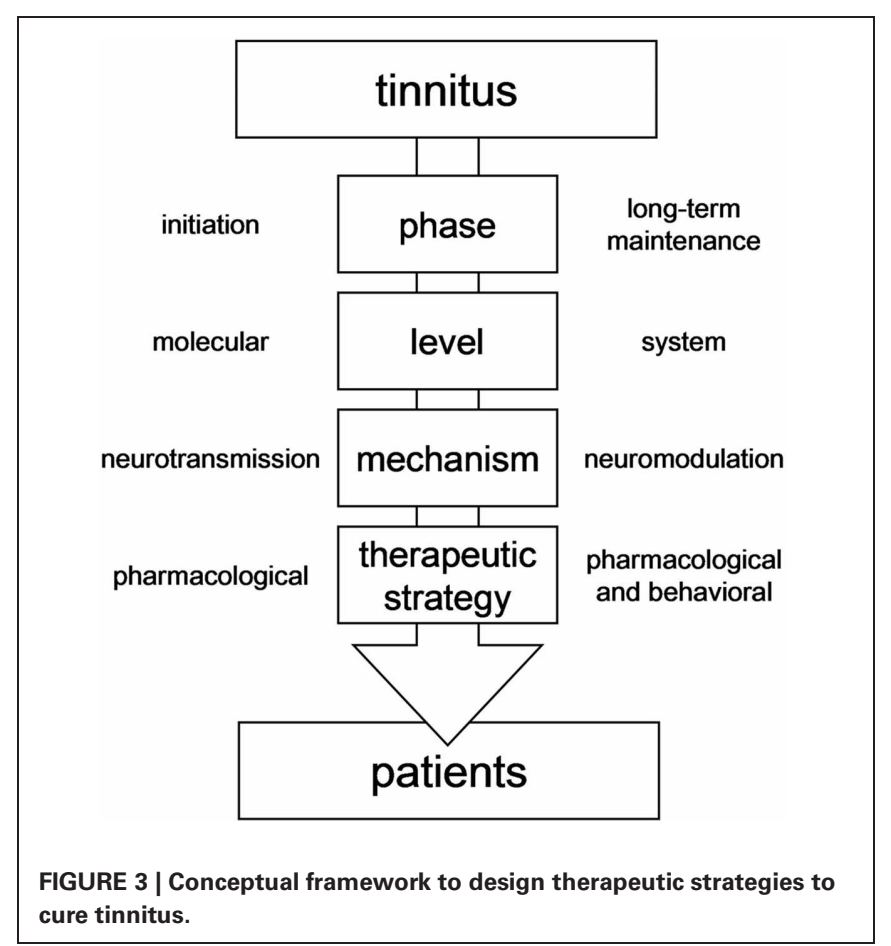


learn to "live with tinnitus." In the context of a pathology which cannot yet be cured, such interventions should not be neglected. The system-level characteristics of tinnitus provide the biological mechanisms to explain how such complementary therapeutic strategies operate.

Understanding of the system dynamics in tinnitus pathophysiology, particularly the limbic component of tinnitus maintenance, allows for the development of more complex combined pharmacological strategies, as suggested earlier (Guitton et al., 2005; Guitton, 2006, 2009). Indeed, one of the most promising ways to cure tinnitus is the development of "hybrid" approaches, combining one specific pharmacological agent aimed at the causal mechanisms of tinnitus and acting on the abnormal neurotransmission in the cochlea with a second less specific pharmacological agent aimed at the symptoms, and regulating the abnormal neuromodulation in centralized neural networks (Figure 3). For instance, an NMDA antagonist locally applied in the cochlea could be used in combination with a drug acting on anxiety levels (Guitton, 2006). In this same line of idea, results obtained in animal suggested that injection of an anxiolytic with action on potassium channels were able to suppress the perception of salicylate-induced tinnitus (Lobarinas et al., 2011). Such molecule, which regulates both neural activity and anxiety levels, could indeed be an interesting candidate for innovative therapeutic strategies.

Understanding the system dynamics can also lead to other non-pharmacological therapeutic strategies. Indeed, the involvement of system dynamics in the maintenance of tinnitus is also what sustains the relative effectiveness of behavioral strategies. In this perspective, the utilization of tele-medicine and virtual reality settings is likely to represent the future of tinnitus-oriented behavioral therapies. Virtual settings, in which multimodality prevails (Guitton, 2010; Lortie and Guitton, 2011), offer indeed new possibilities for behavior-based therapies. The utilization of virtual settings is clearly a way to optimize behavioral therapies. Based on sensory process, tinnitus could be a potential target for behavioral therapeutical strategies based on the use of virtual tools.

\section{REFERENCES}

Apergis-Schoute, A. M., Debiec, J., Doyère, V., LeDoux, J. E., and Schafe, G. E. (2005). Auditory fear conditioning and long-term potentiation in the lateral amygdala require ERK/MAP kinase signaling in the auditory thalamus: a role for presynaptic plasticity in the fear system. J. Neurosci. 25, 5730-5739.

Avan, P., Wit, H. P., Guitton, M., Mom, T., and Bonfils, B. (2000). On the spectral periodicity of transientevoked otoacoustic emissions from normal and damaged cochleas. J. Acoust. Soc. Am. 108, 1117-1127.

Belli, S., Belli, H., Bahcebasi, T., Ozcetin, A., Alpay, E., and Ertem, U. (2008). Assessment of psychopathological aspects and psychiatric comorbidities in patients affected by tinnitus. Eur. Arch. Otorhinolaryngol. 265, 279-285.
Ben Mamou, C., Gamache, K., and Nader, K. (2006). NMDA receptors are critical for unleashing consolidated auditory fear memories. Nat. Neurosci. 9, 1237-1239.

Berman, D. E., and Dudai, Y. (2001). Memory extinction, learning anew, and learning the new: dissociations in the molecular machinery of learning in cortex. Science 291, 2417-2419.

Bledsoe, S. C. Jr., Shore, S. E., and sentation of cortifugal input in the inferior colliculus: a multicontact silicon probe approach. Exp. Brain Res. 221, 530-542.

Casado, M., and Ascher, P. (1998). Opposite modulation of NMDA receptors by lysophospolipids and arachidonic acid: common features with mechanosensitivity. J. Physiol. (Lond.) 513, 317-330. Guitton, M. J. (2003). Spatial repre-

\section{CONCLUSION}

The last decade has witnessed tremendous advances in our understanding of the biological basis of tinnitus. Currently, one of the main challenges in tinnitus research is not necessarily to explore further the biology of tinnitus, but rather to translate these fundamental discoveries into clinical applications. If we succeed in doing so, all-researchers, clinicians, and more importantly the patients-will benefit. Biomedical researchers would see their discoveries applied, the feedback given would help to improve their animal models; the clinicians would finally be able to propose effective therapies to the patients; and the patients would finally have a cure. The present review described some promising ways to treat tinnitus. However, the fact that most evidence are coming from behavioral and anatomical studies rather than clear report of specific synaptic modifications in tinnitus strongly suggest that more work on the synaptic physiology of this pathology is needed.

Given the complexity and the heterogeneity of tinnitus, monofactorial strategies are likely to fail. Rather, a combination of all the possible therapeutic weapons should ideally help us fight this pathology. From a theoretical point of view, tinnitus, as a pathology of synaptic plasticity involving both molecular pathways central for neuronal adaptation, and neuronal networks involved in higher cognitive functions, could represent a powerful model to further our understanding of the remarkable capacity of the brain to adapt to environmental changes and to compute new stimuli.

In conclusion, only a combined understanding of the molecular and of the system-level dimensions of tinnitus will lead to the emergence of innovative and effective therapeutic solutions to help to cure this pathology and provide relief to the sufferers.

\section{ACKNOWLEDGMENTS}

Matthieu J. Guitton holds a Career Grant from the "Fonds de la Recherche en Santé du Québec" (FRSQ). This work was supported by the Canadian Institutes of Health Research (CIHRGrant No. 89699).

Cazals, Y. (2000). Auditory sensorineural alterations induced by salicylate. Prog. Neurobiol. 62, 583-631.

d'Aldin, C. G., Ruel, J., Assié, R. Pujol, R., and Puel, J. L. (1997). Implication of NMDA type glutamate receptors in neural regeneration and neoformation of synapses after excitotoxic injury in the guinea pig cochlea. Int. J. Dev. Neurosci. 15, 619-629.

Dudai, Y. (2004). The neurobiology of consolidations, or, how stable is the engram? Annu. Rev. Psychol. 55, 51-86.

Dudai, Y. (2006). Reconsolidation: the advantage of being refocused. Curr. Opin. Neurobiol. 16, 174-178.

Eggermont, J. J. (2006). Cortical tonotopic map reorganization and its implications for treatment of tinnitus. Acta Otolaryngol. Suppl. 556, 9-12.

Eggermont, J. J., and Roberts, L. E. (2004). The neuroscience of tinnitus. Trends Neurosci. 27, 676-682.

Engineer, N. D., Riley, J. R. Seale, J. D., Vrana, W. A., Shetake, J. A., Sudanagunta, S. P., Borland, M. S., and Kilgard, M. P. (2011). Reversing pathological neural activity using targeted plasticity. Nature 470, 101-104.

Guitton, M. J. (2006). Tinnitus and anxiety: more than meet the ears. Curr. Psychiatry Rev. 2, 333-338.

Guitton, M. J. (2009). Tinnitusprovoking salicylate-treatment triggers social impairments in mice. J. Psychosom. Res. 67, 273-276.

Guitton, M. J. (2010). Cross-modal compensation between name and visual aspect in socially active 
avatars. Comp. Hum. Behav. 26, 1772-1776.

Guitton, M. J., Caston, J., Ruel, J., Johnson, R. M., Pujol, R., and Puel, J. L. (2003). Salicylate induces tinnitus through cochlear NMDA receptors. J. Neurosci. 23, 3944-3952.

Guitton, M. J., and Dudai, Y. (2007). Blockade of cochlear NMDA receptors prevents long-term tinnitus during a brief consolidation window after acoustic trauma. Neural Plast. 2007, 80904.

Guitton, M. J., Klin, Y., and Dudai, Y. (2008). Taste-dependent sociophobia: when food and company do not mix. Behav. Brain Res. 191, 148-152.

Guitton, M. J., and Puel, J. L. (2004). Cochlear NMDA receptors and tinnitus. Audiol. Med. 2, 3-7.

Guitton, M. J., Pujol, R., and Puel, J. L. (2005). m-Chlorophenylpiperazine exacerbates perception of salicylateinduced tinnitus in rats. Eur. J. Neurosci. 22, 2675-2678.

Heffner, H. E., and Harrington, I. (2002). Tinnitus in hamsters following exposure to intense sound. Hear. Res. 170, 83-95.

Heffner, H. E., and Koay, G. (2005). Tinnitus and hearing loss in hamsters (Mesocricetus auratus) exposed to loud sound. Behav. Neurosci. 119, 734-742.

Kaltenbach, J. A., Zacharek, M. A., Zhang, J., and Frederick, S. (2004). Activity in the dorsal cochlear nucleus of hamsters previously tested for tinnitus following intense tone exposure. Neurosci. Lett. 355, 121-125.

Kimura, M., and Eggermont, J. J. (1999). Effects of acute pure tone induced hearing loss on response properties in three auditory cortical fields in cat. Hear. Res. 135, 146-162.

Kossowski, M., Mom, T., Guitton, M., Poncet, J. L., Bonfils, P., and Avan, P. (2001). Fine alterations of distortion-product otoacoustic emissions after moderate acoustic overexposure in guinea pigs. Audiology 40, 113-122.

Ladrech, S., Guitton, M., Saido, T., and Lenoir, M. (2004). Calpain activity in the amikacin damaged rat cochlea. J. Comp. Neurol. 477, 149-160.

Ladrech, S., and Lenoir, M. (2002). Changes in MAP2 and tyrosinated alpha-tubulin expression in cochlear inner hair cells after amikacin treatment in the rat. J. Comp. Neurol. 451, 70-78.
Le Prell, C. G., Yamashita, D., Minami, S. B., Yamasoba, T., and Miller, J. M. (2006). Mechanisms of noiseinduced hearing loss indicate multiple methods of prevention. Hear. Res. 226, 22-43.

Lobarinas, E., Dalby-Brown, W., Stolzberg, D., Mirza, N. R., Allman, B. L., and Salvi, R. (2011). Effects of the potassium ion channel modulators BMS-204352 Maxipost and its R-enantiomer on salicylate-induced tinnitus in rats. Physiol. Behav. 104, 873-879.

Loeb, M., and Smith, R. P. (1967). Relation of induced tinnitus to physical characteristics of the inducing stimuli. J. Acoust. Soc. Am. 42, 453-455.

Lortie, C. L., and Guitton, M. J. (2011). Social organization in virtual settings depends on proximity to human visual aspect. Comp. Hum. Behav. 27, 1258-1261.

Mahlke, C., and Wallhäusser-Franke, E. (2004). Evidence for tinnitusrelated plasticity in the auditory and limbic system, demonstrated by $\arg 3.1$ and c-fos immunocytochemistry. Hear. Res. 195, 17-34.

Milbrandt, J. C., Holder, T. M., Wilson, M. C., Salvi, R. J., and Caspary, D. M. (2000). GAD levels and muscimol binding in rat inferior colliculus following acoustic trauma. Hear. Res. 147, 251-260.

Miller, B., Sarantis, M., Traynelis, S. F., and Attwell, D. (1992). Potentiation of NMDA receptor currents by arachidonic acid. Nature 355, 722-725.

Muluk, N. B., and Oguzturk, O. (2008). Occupational noise-induced tinnitus: does it affect workers' quality of life? J. Otolaryngol. Head Neck Surg. 37, 65-71.

Nicolas-Puel, C., Lloyd Faulconbridge, R., Guitton, M. J., Puel, J. L. Mondain, M., and Uziel, A. (2002). Characteristics of tinnitus and etiology of associated hearing loss: a study of 123 patients. Int. Tinnitus J. 8, 37-44.

Nicoll, R. A., and Malenka, R. C. (1995). Contrasting properties of two forms of long-term potentiation in the hippocampus. Nature 377, 115-118.

Nondahl, D. M., Cruickshanks, K. J., Dalton, D. S., Klein, B. E., Klein, R., Schubert, C. R., Tweed, T. S., and Wiley, T. L. (2007). The impact of tinnitus on quality of life in older adults. J. Am. Acad. Audiol. 18, 257-266.

Noreña, A. J., Gourévitch, B., Aizawa N., and Eggermont, J. J. (2006). Spectrally enhanced acoustic environment disrupts frequency representation in cat auditory cortex. Nat. Neurosci. 9, 932-939.

Ohlemiller, K. K. (2008). Recent findings and emerging questions in cochlear noise injury. Hear. Res. 245 , 5-17.

Panford-Walsh, R., Singer, W., Rüttiger, L., Hadjab, S., Tan, J., Geisler, H. S., Zimmermann, U., Köpschall, I., Rohbock, K., Vieljans, A., Oestreicher, E., and Knipper, M. (2008). Midazolam reverses salicylate-induced changes in brainderived neurotrophic factor and $\arg 3.1$ expression: implications for tinnitus perception and auditory plasticity. Mol. Pharmacol. 74, 595-604.

Peng, B. G., Chen, S., and Lin, X. (2003). Aspirin selectively augmented N-methyl-D-aspartate responses in cultured spiral ganglion neurons of mice. Neurosci. Lett. 343, 21-24.

Puel, J. L., and Guitton, M. J. (2007). Salicylate-induced tinnitus: molecular mechanisms and modulation by anxiety. Prog. Brain Res. 166 141-146.

Reijmers, L. G., Perkins, B. L., Matsuo, N., and Mayford, M. (2007). Localization of a stable neural correlate of associative memory. Science 317, 1230-1233.

Rosenblum, K., Dudai, Y., and RichterLevin, G. (1996). Long-term potentiation increases tyrosine phosphorylation of the N-methyl$\mathrm{D}$-aspartate receptor subunit $2 \mathrm{~B}$ in rat dentate gyrus in vivo. Proc. Natl. Acad. Sci. U.S.A. 93, 10457-10460.

Ruel, J., Chabbert, C., Nouvian, R., Bendris, R., Eybalin, M., Leger, C. L., Bourien, J., Mersel, M., and Puel, J. L. (2008). Salicylate enables cochlear arachidonicacid-sensitive NMDA receptor responses. J. Neurosci. 28, 7313-7323.

Shimizu, E., Tang, Y. P., Rampon, C., and Tsien, J. Z. (2000). NMDA receptor-dependent synaptic reinforcement as a crucial process for memory consolidation. Science 290 , 1170-1174.

Singer, W., Panford-Walsh, R., Watermann, D., Hendrich, O., Zimmermann, U., Köpschall, I.,
Rohbock, K., and Knipper, M. (2008). Salicylate alters the expression of calcium response transcription factor 1 in the cochlea: implications for brain-derived neurotrophic factor transcriptional regulation. Mol. Pharmacol. 73, 1085-1091.

Sotres-Bayon, F., Bush, D. E., and LeDoux, J. E. (2007). Acquisition of fear extinction requires activation of NR2B-containing NMDA receptors in the lateral amygdala. Neuropsychopharmacology 32, 1929-1940.

Suga, N., Gao, E., Zhang, Y., Ma, X., and Olsen, J. F. (2000). The corticofugal system for hearing: recent progress. Proc. Natl. Acad. Sci. U.S.A. 97, 1180-11814.

Takehara-Nishiuchi, K., Nakao, K., Kawahara, S., Matsuki, N., and Kirino, Y. (2006). Systems consolidation requires postlearning activation of NMDA receptors in the medial prefrontal cortex in trace eyeblink conditioning. J. Neurosci. 26, 5049-5058.

Wallhäusser-Franke, E., Mahlke, C., Oliva, R., Braun, S., Wenz, G., and Langner, G. (2003). Expression of c-fos in auditory and nonauditory brain regions of the gerbil after manipulations that induce tinnitus. Exp. Brain Res. 153, 649-654.

Willott, J. F., and Lu, S. M. (1982). Noise-induced hearing loss can alter neural coding and increase excitability in the central nervous system. Science 216, 1331-1334.

Conflict of Interest Statement: The author declares that the research was conducted in the absence of any commercial or financial relationships that could be construed as a potential conflict of interest.

Received: 28 December 2011; accepted: 23 February 2012; published online: 08 March 2012.

Citation: Guitton MJ (2012) Tinnitus: pathology of synaptic plasticity at the cellular and system levels. Front. Syst. Neurosci. 6:12. doi: 10.3389/fnsys. 2012.00012

Copyright (c) 2012 Guitton. This is an open-access article distributed under the terms of the Creative Commons Attribution Non Commercial License, which permits non-commercial use, distribution, and reproduction in other forums, provided the original authors and source are credited. 